International Journal of Computer Networks \& Communications (IJCNC) Vol.3, No.2, March 2011

\title{
Performance InVEstigation On Two-Classes of MANet Routing Protocols aCross VARIOUS MOBILITY MODELS WITH QOS CONSTRAINTS
}

\author{
Foez Ahmed $^{1}$ and Muhammad Sajjadur Rahim ${ }^{2}$ \\ ${ }^{1}$ Dept. of Computer Networks \& Communication Engineering, College of Computer \\ Science, King Khalid University, Kingdom of Saudi Arabia \\ foez28@yahoo.com \\ ${ }^{2}$ Dept. of Information \& Communication Engineering, Rajshahi University, Bangladesh \\ sajid_83@yahoo.com
}

\begin{abstract}
An ad-hoc network is an aggregation of wireless nodes forming a provisional network without any established infrastructure. To facilitate communication within such network, a routing protocol is used to discover and setup routes between nodes. The goal of the routing protocol is to have an efficient route establishment between a pair of nodes, so that messages can be delivered in a timely manner. This research investigates the effects of various mobility models on QoS metrics for two prominent proactive and reactive MANET routing protocols - Fisheye State Routing (FSR) protocol and Ad-hoc On-demand Distance Vector, from Uppsala University (AODVUU) respectively. Performance is measured by the varying number of traffic sources, number of nodes, host velocity, and data sending rate. These two protocols are simulated and compared under different mobility models namely Random Waypoint Mobility Model, Manhattan Grid Mobility Model and Reference Point Group Mobility Model. The performance metrics that are used to evaluate the performance of the routing protocols are Packet delivery ratio (PDR), Average latency of data packets, Network control overhead (NCO) and Throughput. Experimental results reveal that- on-demand routing protocol AODVUU is more sensitive to the speed of mobile nodes than the proactive routing protocol FSR. Due to its proactive nature, FSR can be used in bandwidth and resource critical environment. On the other hand scalability of AODVUU is limited for high-speed network. However, in case of higher data rates AODVUU always performs better in terms of PDR under all three mobility models.
\end{abstract}

\section{KEYWORDS}

MANET, Network Protocols, FSR, AODVUU, Mobility Models, QoS, CBR, NS2.

\section{INTRODUCTION}

Wireless networking is an emerging and increasingly popular technology that allows users to access information and services electronically, regardless of their geographic positions. There are two distinct approaches for enabling wireless communications between mobile hosts. The first approach is to use a fixed network infrastructure that provides wireless access points. The second approach is to form an ad-hoc network among users intending to communicate with each other. This means that all nodes of these networks behave as routers and take part in discovery and maintenance of routes to other nodes in the network. These networks are self-organizing, self-configuring and instantly deployable in response to application needs without a fixed infrastructure. The transmission range of this form of networking is limited by the individual nodes' transmission ranges and is typically smaller compared to the range of cellular systems. 
Ad-hoc networks have several advantages compared to traditional cellular systems. The advantages include 'on-demand' setup, fault tolerance, unconstrained connectivity, ease and speed of deployment and decreased dependency on a fixed infrastructure. Therefore, ad-hoc networks are very attractive for tactical communication in military, law enforcement and disaster relief environments. Not only their importance in military applications is growing, but also their impact on business is increasing. These networks could constitute the infrastructure of numerous applications such as emergency and health-care systems [1], groupware [2], gaming [3] [4] [5], advertisements, customer-to-customer applications (like the UbiBay project [6]) etc. These recent revolutions have been generating a renewed and growing interest in the research and development of MANET.

The routing protocols for ad-hoc networks have to adapt quickly to the frequent and unpredictable changes of topology and must be parsimonious of communications and processing resources. The movement of network's constituents characterizes mobile systems and due to the host mobility, network topology may change from time to time. Therefore, it is critical and more challenging for the routing protocols to deliver data packets efficiently between source and destination. Existing MANET routing protocols are typically subdivided into two main categories [7] according to their design philosophy: (a) proactive routing protocols and (b) reactive/on-demand routing protocols. Proactive routing protocols are derived from legacy Internet distance-vector [8] and link-state [9] protocols. They attempt to maintain consistent and updated routing information for every pair of network nodes by propagating, proactively, route updates at fixed time intervals. Reactive on demand routing protocols, on the other hand, establish the route to a destination only when there is a demand for it. The source node through the route discovery process usually initiates the route requested. Once a route has been established, it is maintained until either the destination becomes inaccessible (along every path from the source), or until the route is no longer used, or expired.

At the same time, Quality of Service (QoS) models in ad-hoc networks become more and more required because more and more real time applications are implemented on the network. Moreover, an ad-hoc network must supply a wide category of services e.g. group calls, situation awareness data, and Internet connections. The different services can have different Quality of Service (QoS) demands. In routing layer, QoS are guaranteed in terms of data rate, packet delivery ratio, control packet overhead, delay variance (jitter) and so on. Hence, an important component in providing these services is the routing protocol, i.e. the protocol that finds and determines by which route through the network a packet should be forwarded on its way to its destination. For a protocol to provide good QoS it must determine new routes rapidly with minimal bandwidth consumption. Using a protocol that provides good quality of service will greatly affect the MANET's performance. A MANET QoS framework must be able to find multi-hop paths with sufficient bandwidth and delay characteristics, despite network changes, low bandwidth links and shifting traffic patterns.

In reality, the performance of mobile ad-hoc networks will depend on many factors such as node mobility model, traffic pattern, network topology, radio interference, obstacle positions, and so on. Most of early simulation works used only one mobility model, Random Waypoint [10] [11] to evaluate ad-hoc routing protocols. This model is widely used in most simulation studies. However, it has been shown that mobility pattern plays an important role in the performance of ad-hoc routing protocols. The same protocol can perform differently in different mobility patterns [12] [13]. Random Waypoint represents typical random node movement pattern but could be unrealistic in many real situations. A few different mobility models have been developed to model different ad-hoc network environments other than entirely random movements. Reference Point Group Mobility Model (RPGM) [14] is used to describe scenarios where users move in groups. It is very useful to model events such as conference, art gallery exhibition, tourist groups, searching rescue mission and military actions. Manhattan Grid [15] is another model for describing user movement in downtown area where street topology is gridlike, as in Manhattan of New York City. 
International Journal of Computer Networks \& Communications (IJCNC) Vol.3, No.2, March 2011

\section{RELATED WORKS}

Broch et al., In [16] compared the four ad-hoc routing protocols (AODV, DSR, TORA, and DSVD) in terms of packet delivery ratio, routing overhead, and path optimality. This work provides detailed performance analysis on Ad-hoc routing protocols but only Random Waypoint mobility model was used.

In [17], the authors introduce a novel proactive (FSR) routing scheme, which is adapted to the wireless ad-hoc environment. They compare the performance of FSR routing protocol with on demand routing protocols such as AODV and DSR and their simulation experiments show that FSR is a simple, efficient and scalable routing solution in a mobile, ad-hoc environment. They also conclude that FSR is more desirable for large mobile networks where mobility is high and the bandwidth is low. However, all the performances are measured under only a single mobility model: Random Waypoint model.

In [18] the authors do not evaluate the protocols in extreme mobile environment (speed is constrained to $0-10 \mathrm{~m} / \mathrm{s}$ ). The protocols examined are WRP, FSR and AODV, with CSMA, MACA, FAMA and 802.11 DCF.

The paper, [19] introduces a new protocol WRP-Lite, which is a table-driven routing protocol and performs comparative evaluations with existing protocol - dynamic source routing (DSR) protocol. The authors evaluate the performance of WRP-Lite and DSR for varying degree of mobility and traffic in a 20-node network. From simulation they observed that WRP-Lite has much better delay and hop performance while having comparable overhead to DSR.

In [20], a comparative evaluation of proactive and reactive routing protocols (DSR, AODV, FSR, WRP [21], LAR [22], DREAM [23] is presented. A variety of scenarios with independent and group mobility is employed. They classify protocols according to their performance and assay their suitability for various usage modes. Mobility ranges from 0 to $70 \mathrm{~km} / \mathrm{h}$. They conclude that no protocol is universally the best choice and protocol selection should be based on the usage mode.

Another performance comparison was done by [24]. In this work AODV, DSR, TORA and DSDV are compared under three different mobility models: Random Waypoint, RPGM, and Manhattan Grid and various scenarios and focuses on power consumption issue. They conclude that the performance of ad-hoc routing protocols greatly depends on the mobility model it runs over and reactive protocols are more speed-sensitive while proactive protocols are not. They also conclude that on-demand protocols perform better than proactive ones in terms of energy conservation.

AODV and OFLSR, with most of the proposed optimizations are compared in [25]. They study the impact of routing protocols for wireless mesh network and infer that under increased traffic load and mobility, the selected on-demand routing protocol like AODV is not scalable. On the contrary, their proposed table-driven routing protocol OFLSR always provides a better performance in terms of data packet delivery ratio, throughput, packet latency and routing overhead, under different traffic and mobility instances. They consider both TCP and CBR traffic sources and investigate the performance of these two protocols across random waypoint mobility model only.

[26], evaluates the performance of ad-hoc unicast routing protocols under extreme mobility (over $20 \mathrm{~m} / \mathrm{s}$ ). They present extensive evaluation of two reactive (DSR and AODV) and one proactive (FSR) protocol under Glomosim's statistical propagation models for multipath fading. Their simulation results infer that at very high speeds DSR is inferior to AODV and FSR. AODV exhibits impressive resilience to mobility and it is suitable for use in highly dynamic environments, whereas FSR maintains sufficient reliability for increased mobility. The performance evaluation of AODVUU and Destination-Sequenced Distance-Vector Routing 
(DSDV) combined with TCP Newreno, TCP Vegas and TCP AP was done by [27]. The simulation-based evaluation concluded that the best performance was achieved with TCP Vegas in conjunction with AODVUU.

The rest of the paper is organized as follows. Section 3 provides a brief overview of MANET routing protocols. The simulation environment together with simulation parameters are presented in section 4 . Section 5 analyses the simulation results elaborately in terms of graphs and provides comments on the basis of analysis. Finally, the concluding remarks and the recommendations for future work in this area are provided in section 6 .

\section{Brief Overview of Manet Routing Protocols}

\subsection{Fisheye State (FSR) Routing Protocol}

The goal of Fisheye State Routing (FSR) protocol is to provide an accurate routing solution while the control overhead is kept low. The FSR concept originates from Global State Routing (GSR) [28]. GSR can be viewed as a special case of FSR, in which there is only one fisheye scope level. However, this scheme uses the "fisheye" technique proposed by [29], where the technique was used to reduce the size of information required to represent graphical data. This protocol is widely studied in [17] [30] [31] and named "Fisheye Routing" due to the novel 'fisheye' updating mechanism. FSR is functionally similar to Link State (LS) Routing in that it maintains a topology map at each node. The key difference is the way in which routing information is disseminated. In LS, link state packets are generated and flooded into the network whenever a node detects a topology change. In FSR, link state packets are not flooded. Instead, nodes maintain a link state table based on the up-to-date information received from neighboring nodes, and periodically exchange it with their local neighbors only (no flooding). So, each node gets accurate information about neighbors and the detail and accuracy of information decreases as the distance from the node increases. However, the following properties of FSR highlight its importance in MANETs.

- Simplicity

- Usage of up-to-date shortest routes

- Robustness to host mobility

- Exchange partial routing update with neighbors

- Reduced routing update traffic

\subsubsection{Data Structure}

Each node running FSR has to maintain a neighbor list, a topology table, and a routing table. In the neighbor list, the node keeps the addresses of all nodes one hop away and the time they it received the last information from that neighbor. If the node does not receive any link state information from a neighbor for a certain interval, the neighbor is removed from the neighbor list. In the topology table, information about all destinations in the network is stored. From this table, the node creates the routing table used for routing data messages. The topology table is updated through update messages sent from neighbors which will be discussed in the next section.

\subsubsection{Sending Update Message}

To obtain lower levels of overhead traffic in a mobile network, the generation of update messages is not event-driven, but periodic. When a node obtains a new neighbor or loses one, it updates its neighbor list, topology table, and routing table. To reduce the amount to traffic that is transmitted, the node only includes information about nodes with certain periodicities and if the "Need To Send" (NTS) flag is set to true. Furthermore, the node sets the NTS flag to true for the entry in the topology table that contains the node's own topology data with the periodicity $T_{U}$ regardless whether any of the node's neighbors have changed. 


\subsubsection{Receiving Update Messages}

When a node receives an update information message, it first checks whether the sender is a neighbor and if it is included in the neighbor list. New neighbors are added, and the time the node last received an update message from the neighbor is updated. Then the topology table is updated with the information. The contents of all entries in the packet are studied. If it is a new destination, a new topology table entry is created, and the NTS flag is set, since this information needs to be forwarded. Otherwise, if the entry already exists in the topology table, the sequence numbers are checked and compared. If the incoming entry has a larger sequence number than the existing number, the information has changed, and the entry in the table needs to be updated. The "Need To Send" (NTS) flag is set since the information is new.

If, instead, the sequence number is lower, this means that the sender is not updated with the latest information. No changes are made in the topology table but the NTS flag is set and the information is forwarded. If the sequence number is equal to the previous number nothing happens. When new information has been added to the topology table, the routing table is also updated with the most recent information about routes in the network. Moreover, information is discarded when it is too old.

\subsection{AODV-UU (Ad-hoc On-demand Distance Vector, From Uppsala University) Routing Protocol}

AODV-UU is a routing protocol under investigation by the IETF for use in ad-hoc networks, where both end-users and routers are mobile. This implementation supports IPv6 and multicasting (with the appropriate patch) and is compliant with RFC 3561 [32]. AODV-UU is a Linux implementation of the AODV (Ad-hoc On-demand Distance Vector) [33] routing protocol, developed at Uppsala University, Sweden. AODV-UU implements all mandatory and most optional features of AODV.

\subsubsection{Packet handling and Packet arrival}

AODV-UU uses Netfilter - a software framework, for all its packet processing and modification needs. When a packet traverses the protocol stack, it is caught by the Netfilter hooks that have been set up by the AODV-UU kernel module, kaodv. The nf_aodv_hook() function of the kaodv module identifies the packet type, and either tells Netfilter to accept the packet (i.e., to let it through and allow the system to process it on its own) or to queue it (for further processing by AODV-UU in user-space). Non-IP packets are always accepted, since these packets are of no interest to AODV-UU. Locally generated packets are always queued, since a route may have to be determined for those. Incoming AODV control messages are always accepted, since these eventually should be processed on a separate UDP socket and must be let through in order to be able to arrive there. Also, only packets on AODV-UU-enabled network interfaces should be processed by AODV-UU. Packets on other network interfaces are therefore immediately accepted, and not processed further.

\subsubsection{Data packet processing}

If the destination of the packet (determined by its destination IP address) is the current host, the packet is a broadcast packet, or Internet gateway mode has been enabled and the packet is not a broadcast within the current subnet, the packet is accepted. This means that the packet under these circumstances will be handled as usual by the operating system. Otherwise, the packet should either be forwarded, queued or dropped. The internal routing table of AODV-UU is used for checking whether an active route to the specified destination exists or not. If such a route exists, the next hop of the packet is set and the packet is forwarded. Otherwise, provided that the packet was generated locally, it is queued at the source node until AODV-UU has decided on an action, and a route discovery is initiated. If the packet was not generated locally, and no route was found, it is instead dropped and a RERR message is sent to the source of the packet. 


\section{Simulation ToOLS}

For the design and implementation of communication protocols and algorithms, the use of simulation tools provides a substantial productivity and allows near perfect experimental control in various wireless networks.

\subsection{Simulation Environment}

The simulation experiments were conducted using the network simulator NS2 [34]. In this experiment, the distributed coordination function (DCF) of standard IEEE 802.11 (IEEE Computer Society, 1997) is used as the MAC layer protocol. It uses Request-to-Send (RTS) and Clear-To-Send (CTS) control packets to provide virtual carrier sensing for unicast data packets to overcome the well-known hidden terminal problem. The simulated radio interface model is the Lucent WaveLAN [35], which is modeled as shared-media radio with an 802.11 DSSS. As signal propagation model, we use the "Shadowing Model", which is a more realistic model taking into account multi-path propagation effects, i.e., fading. The transmission range for each node is $250 \mathrm{~m}$ with channel capacity of $2 \mathrm{Mbits} / \mathrm{sec}$ and the carrier sensing range is $471.5 \mathrm{~m}$.

All simulations are performed with varying nodes $(10 \sim 70)$ in a rectangular area of $670 \times 670$ meter $^{2}$ where the duration of each simulation is 200 seconds. All protocols use an interface queue (IFQ) of 50 packets. The IFQ is a FIFO priority queue where routing packets get higher priority than data packets. All MAC and Network layer operations of the wireless network interfaces are logged in trace files. Post simulation analyses are performed to each of the trace files by using parsing language in order to calculate the value of PDR, Throughput, NCO and Average latency.

The source-destination pairs are randomly selected among all nodes in the network. To provide traffic load to the ad-hoc network, constant bit rate (CBR) traffic sources are used in our simulation study. For each CBR session, the data payload size is 512 bytes and packet rates are varied from $2 \mathrm{pkts} / \mathrm{sec}$ to $10 \mathrm{pkts} / \mathrm{sec}$ due to different simulation scenarios.

BonnMotion [36] is used to generate movement patterns for all mobility models: Manhattan Grid, Random Waypoint and RPGM. To see the impact of node mobility on the network performance, we change the average speed of the mobile nodes in each mobility scenario. We consider four speed levels: $1 \mathrm{~m} / \mathrm{s}$ (Walk), $5 \mathrm{~m} / \mathrm{s}$ (Bicycle), $10 \mathrm{~m} / \mathrm{s}$ (Motorcycle), and $15 \mathrm{~m} / \mathrm{s}$ (Car) for our simulation experiments that were used by [37].

\subsection{Measurement parameters}

The performance of routing protocols (FSR and AODVUU) are compared using the following important Quality of Services (QoS) metrics:

1. Packet Delivery Ratio (PDR): Packet delivery ratio is an important metric as it describes the loss rate that will be seen by the transport protocols, which run on top of the network layer. Thus packet delivery ratio in turn reflects the maximum throughput that the network can support. It is defined in [38][39] as the ratio between the number of packets originated by the application layer CBR sources and the number of packets received by the CBR sink at the final destination.

$$
\mathrm{PDR}=\frac{\sum_{1}^{N} C B R_{\text {recv }}}{\sum_{1}^{N} C B R_{\text {Send }}}
$$

where $\mathrm{N}$ is the number of data sources, $\mathrm{CBR}_{\text {recv }}$ is the total number of CBR packets received and $\mathbf{C B R}_{\text {Send }}$ is the total number of CBR packets sent per source. 
2. Average Latency: This includes all possible delays caused by buffering during route discovery, queuing delay at the interface, retransmission delays at the MAC, propagation and transfer times. The lower the packet latency the better the application performance as the average end-to-end delay is small. According to [38], once the time difference between every CBR packet sent and received was recorded, dividing the total time difference over the total number of CBR packets received gave the delay for the received packets and defined as:

$$
\text { Average Latency }=\frac{\sum_{1}^{N}\left(C B R_{\text {sendTime }}-C B R_{\text {recvTime }}\right)}{\sum_{1}^{N} C B R_{\text {recv }}}
$$

where $\mathrm{N}$ is the number of data sources.

3. Network Control Overhead (NCO): Network control overhead is used to show the efficiency of the MANET's routing protocol scheme. It is defined, in [39], as the ratio of the number of control messages (the number of routing packets, Address Resolution Protocol (ARP), and control packets e.g., RTS, CTS and ACK) propagated by each node throughout the network and the number of the data packets received by the destinations. The definition is:

$$
\mathrm{NCO}=\frac{\text { Number of Control Message Sent }}{\text { Number of data Received }}
$$

4. Throughput: This is the measure of how soon an end user is able to receive data packet. It is determined as the ratio of the total data received by the end user and the connection time [37]. A higher throughput will directly impact the user's perception of the quality of service (QoS).

$$
\text { Throughput }=\frac{\text { Total Received Packets }}{\text { Total Simulation Time }} \quad(\mathrm{kbits} / \mathrm{sec})
$$

\section{Simulation ReSUlts ANAlysis AND Discussion}

This section presents the results obtained from the simulations. For the fairness of protocol comparison and network performance, each ad-hoc routing protocol is run over the same set of scenarios.

In the graphs the legend data will be interpreted as follows: If the graph is for Data sending rate Vs Packet Delivery Ratio (PDR) then the legend data should be interpreted as follows:

1. FSR-RW: means the value of PDR of FSR routing protocol in the Random Waypoint (RW) Mobility Model.

2. AODVUU-RW: means the value of PDR of AODVUU routing protocol in the Random Waypoint (RW) Mobility Model.

3. FSR-MH: means the value of PDR of FSR routing protocol in the Manhattan (MH) Grid Mobility Model.

4. AODVUU-MH: means the value of PDR of AODVUU routing protocol in the Manhattan (MH) Grid Mobility Model.

5. FSR-RM: means the value of PDR of FSR routing protocol in the Reference Point Group Mobility Model (RM).

6. AODVUU-RM: means the value of PDR of AODVUU routing protocol in the Reference Point Group Mobility Model (RM).

The same convention is followed for all other graphs. In place of Packet Delivery Ratio (PDR), Network Control Overhead (NCO), Average Latency, and throughput will occur in various graphs. 


\subsection{Effect of the Speed of Nodes on QoS Metrics across Three Mobility Models}

To evaluate the effect of the speed of nodes on QoS metrics, the simulation is run for each of the routing protocols where total numbers of nodes are 30, maximum connections among nodes are 20 , pause time is 0 second and maximum data rate is 4 pkts/sec. The simulation results are plotted in Figure 1, Figure 2 and Figure 3 for Random Waypoint Mobility Model, Manhattan Grid Mobility Model, and Reference Point Group Mobility Model respectively.
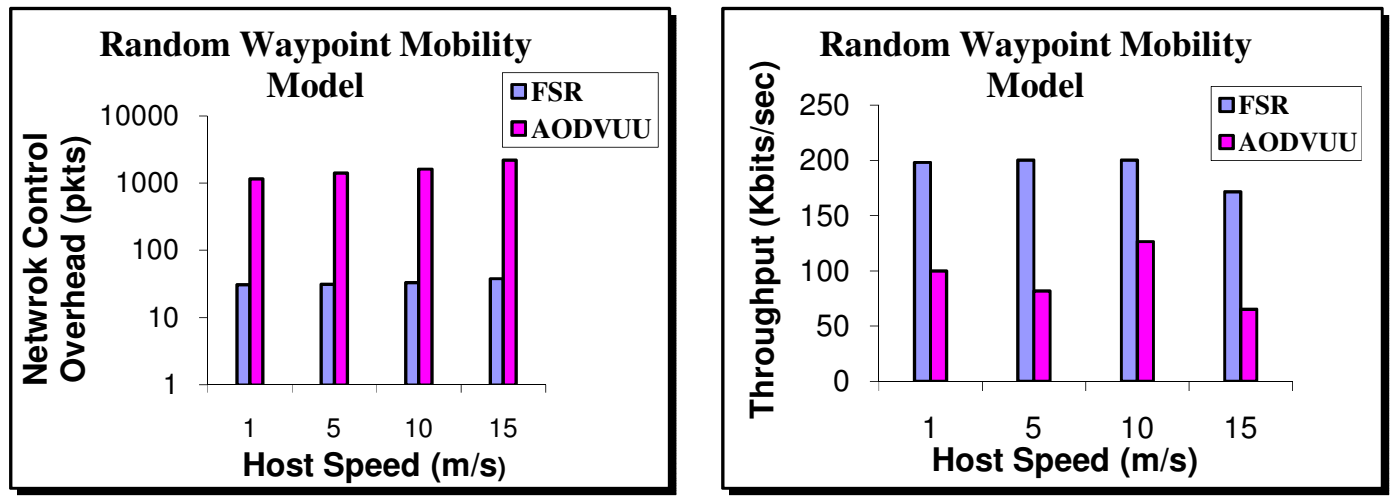

Figure 1. (a) Network Control Overhead and (d) Aggregate CBR throughput Vs Host Mobility (Speed) across Random Waypoint mobility model
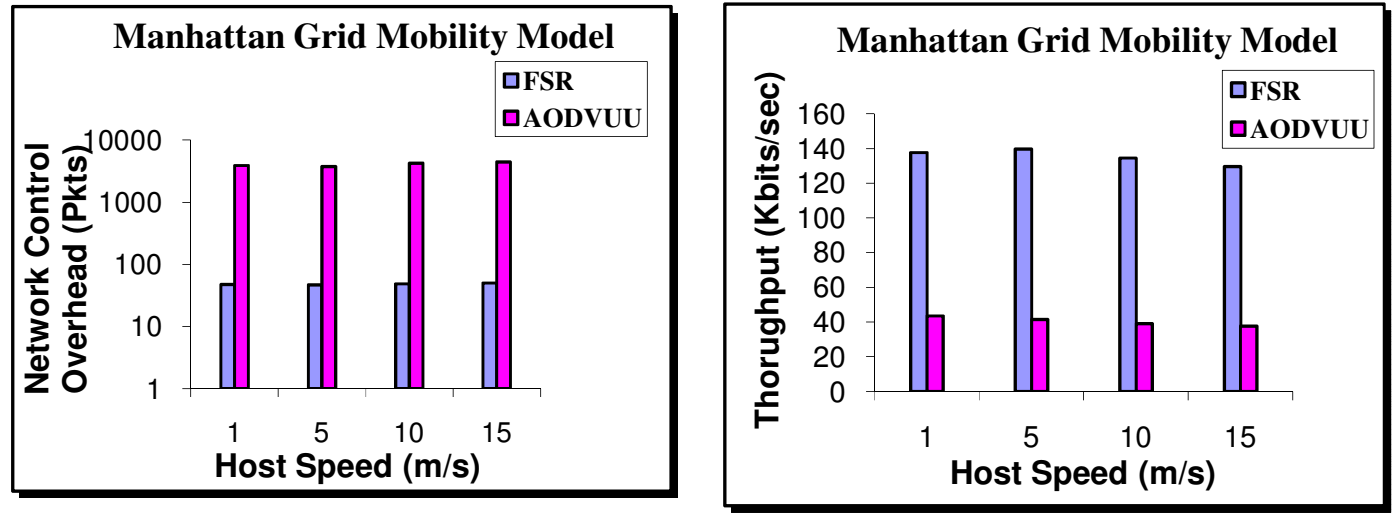

Figure 2. (b) Network Control Overhead and (e) Aggregate CBR throughput Vs Host Mobility (Speed) across Manhattan Grid Mobility Model
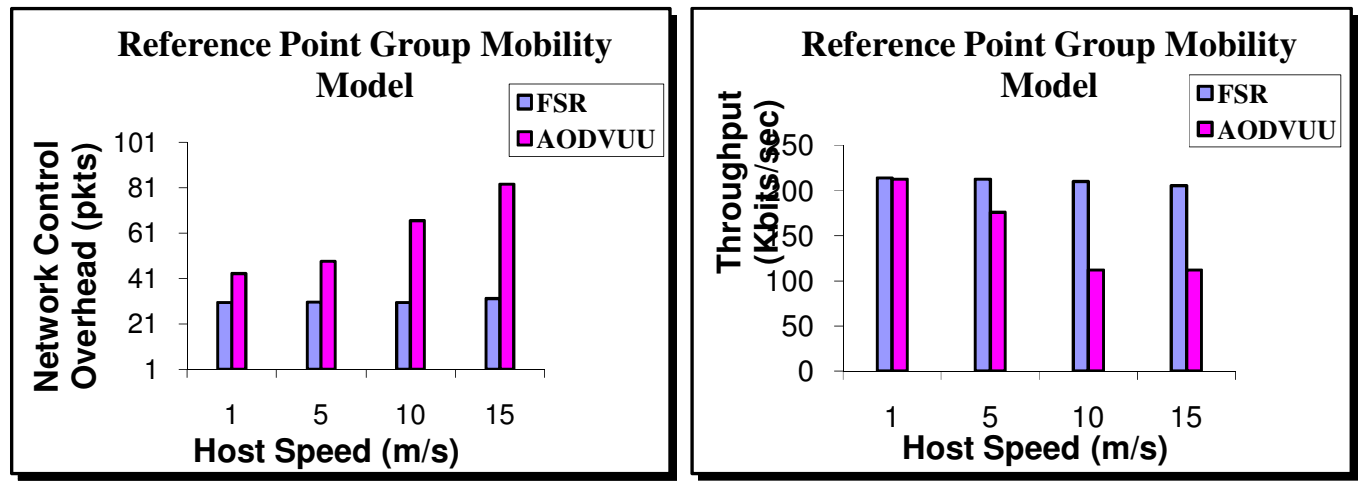

Figure 3. (c) Network Control Overhead and (f) Aggregate CBR throughput Vs Host Mobility (Speed) across Reference Point Group Mobility Model 
From these graphs, it is observed that the network control overhead for both FSR and AODVUU increases with increasing mobility. At lower speed AODVUU performs better than that of higher speed across all the three mobility models. This is because at lower speed, the topology changes are less frequent and hence AODVUU sends less routing and control messages. However, as speed goes up, route changes are made more frequently and it initiates route discovery mechanism in AODVUU. Therefore, to build up new routes, AODVUU has to generate more control overhead, which consumes more bandwidth.

It is also noticeable that FSR exhibits an order of magnitude smaller control overhead than that of AODVUU due to its proactive nature. Therefore, FSR demonstrates sufficient reliability mainly due to the mobility based interval adjustment. However, the frequency with which FSR exchanges link state information is proportional to mobility, therefore control overhead increased with speed but not sharply. This result shows that on-demand protocols are more sensitive to speed of mobile nodes than proactive protocols.

Also from the entire graph it is observed that the Manhattan grid - like street environment is more challenging for both FSR and AODVUU than the other two environments. In Manhattan Grid model, more network control overhead is needed to route the data packets, which consumes more bandwidth. On the other hand, both FSR and AODVUU, specially FSR protocol is more scalable in Reference Point Group mobility environment. This is because for both cases nodes move in groups and this pattern reduces the rate of topology changes. This reduction of topology change is directly reflected in the amount of bandwidth consumed by each protocol. Thus, having proactive nature of FSR, this protocol can be used in bandwidth and resource critical environment. On the other hand scalability of AODVUU is limited for highspeed network.

The results for throughputs for FSR and AODVUU are also plotted in Figure 1(d), Figure 2(e) and Figure 3(f), which is consistent with the control overhead shown in Figure 1(a), Figure 2(b) and Figure 3(c) respectively. This is because the growth of overhead is directly related to the throughput drop. Results show that the throughput of AODVUU degrades quickly when the mobility increases, no matter which mobility model is used. AODVUU's poor performance is due to heavy network control message overhead and hence the superiority of FSR is remarkable. Again the throughput of FSR is sufficiently higher across Reference Point Group mobility environment than other two mobility models. This is because the hierarchical structure of FSR can match well the group motion. The throughput of group mobility model does not decrease too drastically when the mobility increases shown in Figure 3(f). This stability comes from the hierarchical routing structure. In terms of throughput, it is also noticed that the Manhattan Grid - like street environment can be more challenging than the other two mobility models.

\subsection{Effect of Data Sending Rate on QoS Metrics across Three Mobility Models}

To evaluate the effect of Data sending rate on QoS metrics, the simulation is run for each of the routing protocols where total number of nodes are 50, pause time is 10 seconds, maximum speed of nodes is $7 \mathrm{~m} / \mathrm{s}$ and connection pairs among nodes are 20 . The simulation results are plotted in Figure 4 through Figure 7. 


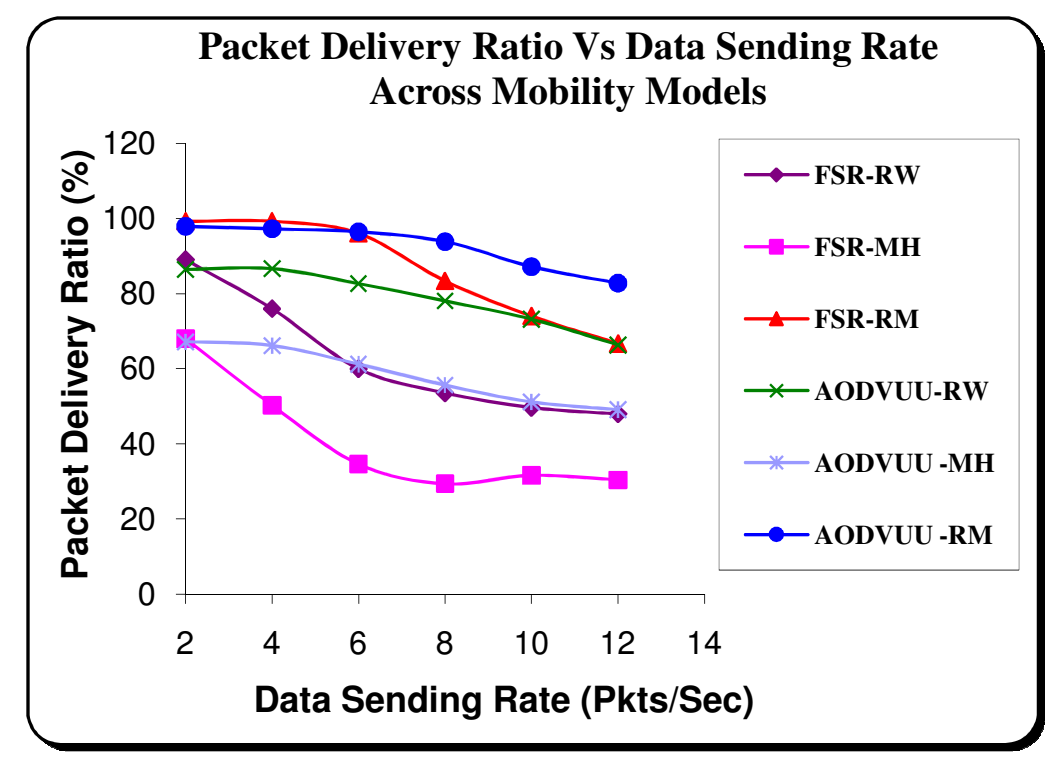

Figure 4. Packet Delivery Ratio Vs Data Sending Rate across Three Mobility Models

From figure 4 it is observed that at low data rate the packet delivery ratio (PDR) for FSR is higher than that of AODVUU in all three mobility models and its value varies from $68 \%$ to $100 \%$. At high data rates AODVUU always performs better in terms of PDR than that of FSR. It is also seen that the packet delivery ratio decreases whenever the data rate increases, no matter which mobility model is used. The reason is that as the data rate is increased the more data is injected into the network before processing the previous data, which overwhelms the queue, leading to the increase in the network congestion, and hence more data packets are lost.

From the graph, it is also seen that the value of packet delivery ratio (PDR) for both FSR and AODVUU protocol degrades more drastically in Manhattan Grid-like urban environments. On the other hand, these two protocols are more scalable in Reference Point Group mobility model - like military or rescue operation scenarios. At moderate data rate FSR always performs better than that of AODVUU in terms of PDR because a full topology map is kept at each node and shortest paths are computed using this map.

In Figure 5, the average latency (msec) of FSR and AODVUU across three mobility models is reported. Here it is observed that for both of the two protocols, the average latency goes up rapidly as the data rate is increased no matter which environment is used. In terms of latency, AODVUU out performs FSR at low data rate as well as high data rate. The reason is that the FSR employs a link-state routing algorithm. Link-state requires more computation complexity than Distance-vector (where AODVUU uses distance-vector algorithm partially), hence linkstate requires more computation steps for a node to perform routing computations form the update messages. 


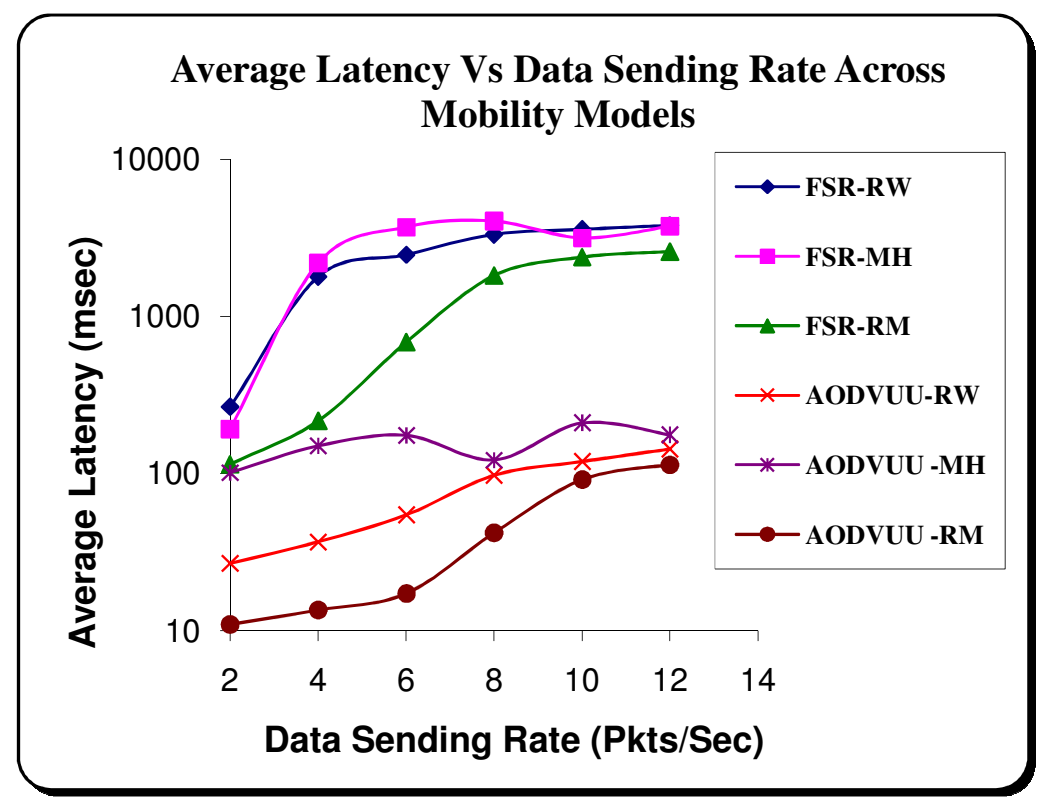

Figure 5. Average Latency Vs Data Sending Rate across Three Mobility Models

In Reference Point Group mobility model, both the protocols need minimum latency to compute optimum route than other two models at lower data rate. The increase of latency of FSR in Reference Point Group mobility model is not too sharp as like other two models. Whereas Manhattan grid - like street environment is more challenging environment for both reactive and proactive routing protocols, especially for FSR while considering average data sending rate (say $8 \mathrm{Pkts} / \mathrm{Sec})$. In Figure 5, it is also observed that at low data rate (2 pkts/sec), the latency of FSR in Reference Point Group mobility model and the latency of AODVUU in Manhattan grid mobility model is almost same.

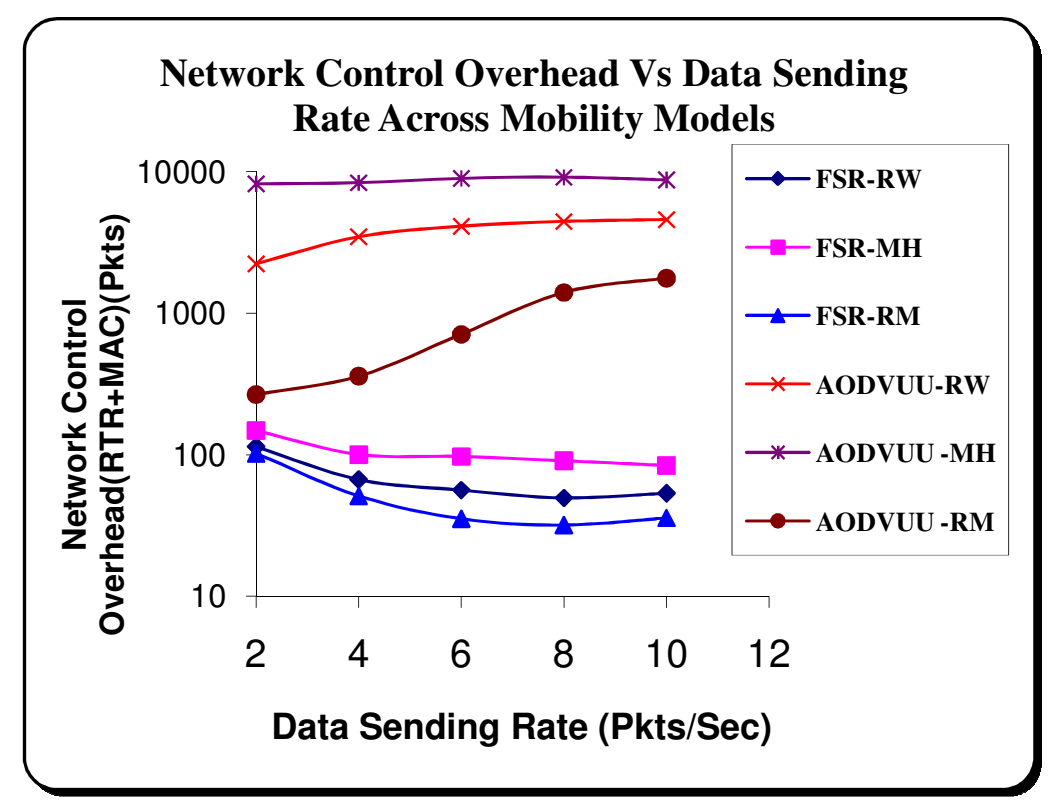

Figure 6. Network Control Overhead Vs Data Sending Rate across Three Mobility Models 
From Figure 6, it is observed that the network control overhead needed for FSR routing protocol goes down as the value of data rate increases. This occurs because nodes wait a longer time before transmitting Link State Packets (LSPs) it received from other nodes at each successive scope. This results in lower control overhead traffic. The reductions of network control overhead at higher data rate are very significant. This is because the same amounts of routing and control message are needed to route CBR traffic at lower data rate as well as at higher data rate.

But, here the exception is remarkable for AODVUU routing protocol, which is reported in Figure 6. The network control overhead is increased as the data rate is increased. The control overhead of AODVUU is higher than that of FSR. It is also noted that the Manhattan Grid mobility - like environment is more challenging for both on-demand and proactive routing protocol where these two protocols are more scalable in Reference Point Group mobility model in terms of network control overhead. Here it is also noticeable that the FSR is more optimum in any environment than that of AODVUU.

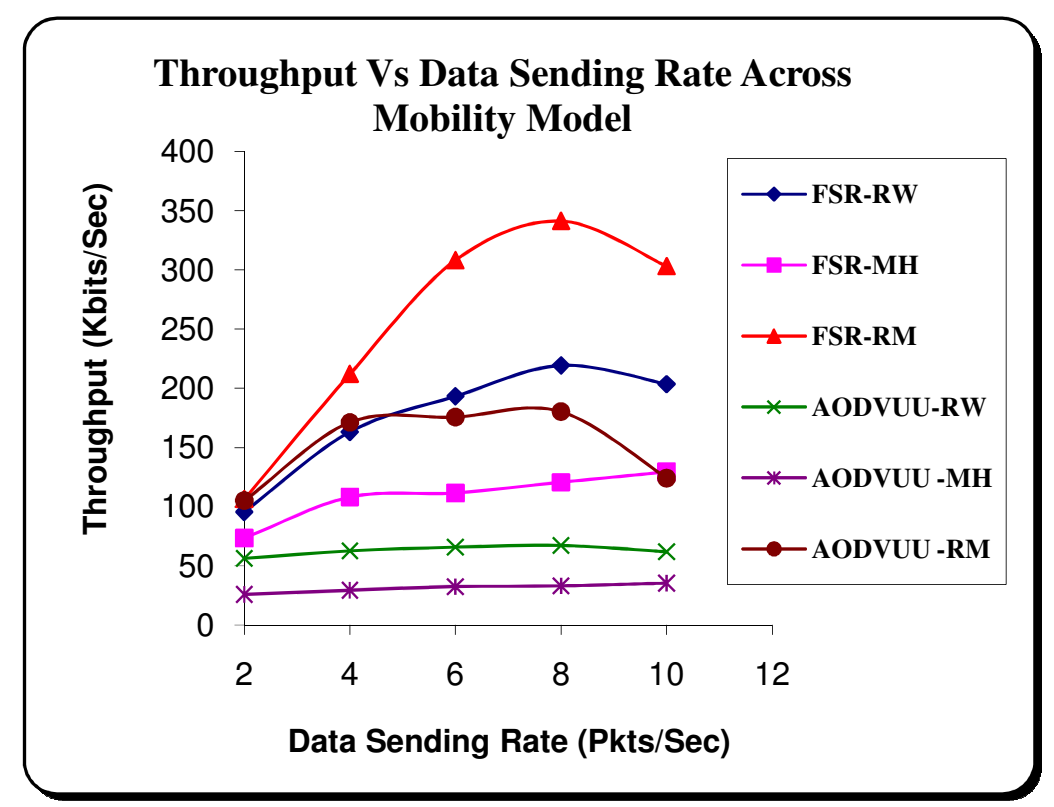

Figure 7. Throughput Vs Data Sending Rate across Three Mobility Model

The throughput (Kbits/sec) of FSR and AODVUU across three mobility models is presented in Figure 7. Form graph, it is observed that the throughput for FSR protocol is higher than that of AODVUU protocol in all three mobility models. The throughput increases as the data rate increases but at very high data rate the throughput for both of the two protocols decreases no matter which mobility model is used. At very high data rate the more amounts of data traffics are injected into the network, which overwhelms the queue, and hence more data packets are lost which degrades throughput. The throughput for FSR varies from $73 \mathrm{Kbits} / \mathrm{sec}$ to 106 $\mathrm{Kbits} / \mathrm{sec}$ while for AODVUU it varies from $25 \mathrm{Kbits} / \mathrm{sec}$ and $105 \mathrm{Kbits} / \mathrm{sec}$ at low data rate. From Figure 7, it is also observed that the throughput for FSR in Reference Point Group mobility model increases more sharply at moderate data rate (e.g. 4 or $6 \mathrm{Pkts} / \mathrm{sec}$ ) than other mobility models while the throughput for AODVUU goes up slowly with increased data rate. Obviously, FSR is more scalable for group mobility model- like military, rescue operation environments and so on where throughput is more demandable. 


\subsection{Effect of Number of Nodes on QoS Metrics across Three Mobility Models}

To evaluate the effect of number of nodes (dense network) on QoS metrics, the simulation is run for each of the routing protocols where maximum connections are 7, pause time is 0 seconds, maximum speed of nodes is $7 \mathrm{~m} / \mathrm{s}$ and maximum data rates is $4 \mathrm{pkts} / \mathrm{sec}$. The simulation results are plotted in Figure 8 through Figure 11.

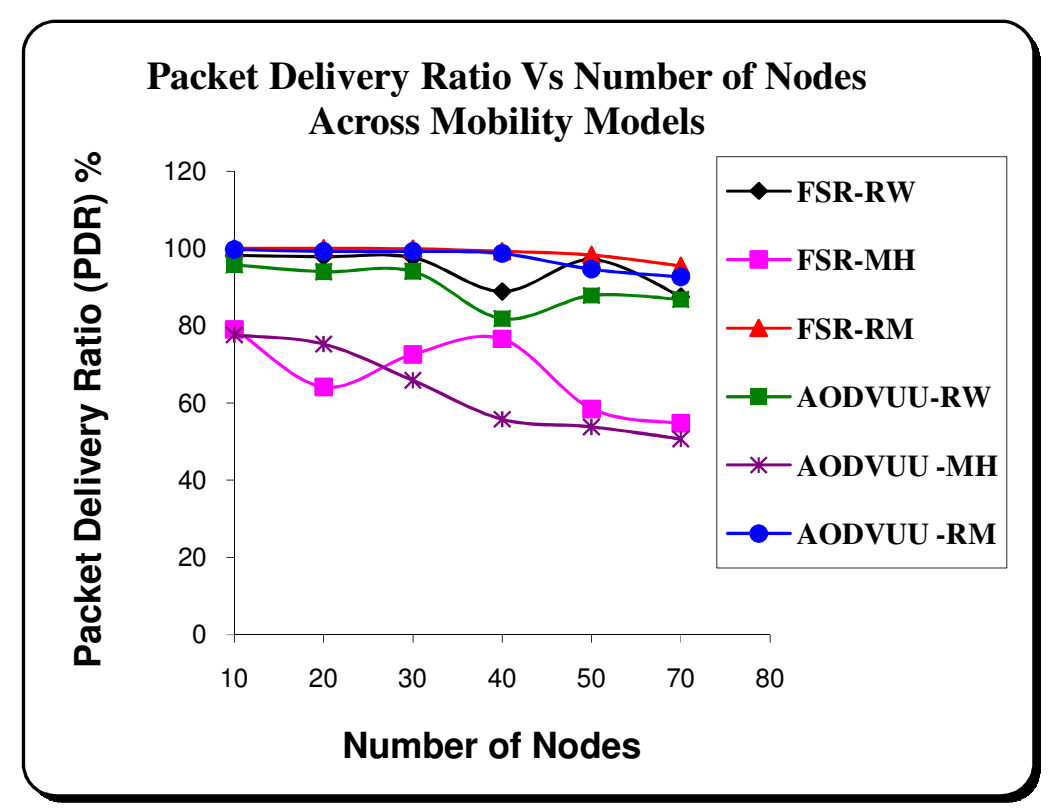

Figure 8. Number of Nodes Vs Packet Delivery Ratio Across three Mobility Models

Figure 8 shows the effect of packet delivery ratio (PDR) as a function of number of hosts for FSR and AODVUU routing protocol across the three mobility models. The packet delivery ratio for both of the two protocols goes down when the number of mobile hosts is increased no matter which mobility model is used. However, the packet delivery ratio of FSR degrades gracefully for the increasing host numbers than that of AODVUU. This infers that the FSR protocol works most efficiently in the dense network while AODVUU does not.

From Figure 8, it is also observed that both of the two protocols are more scalable in the Reference point group mobility model than other two mobility models. The Manhattan grid mobility model - like urban environment is more challenging for these two protocols especially for AODVUU while considering the performance of packet delivery ratio (PDR).

Figure 9 shows the simulation results of average latency as a function of number of hosts for FSR and AODVUU routing protocol across the three mobility models. It is observed that the average latency for FSR and AODVUU increases when the number of hosts increases under all the three mobility models. AODVUU outperforms FSR in terms of latency. It is also noticeable that at Manhattan grid mobility model FSR performs well than AODVUU while considering the number of hosts is about up to 40 . 


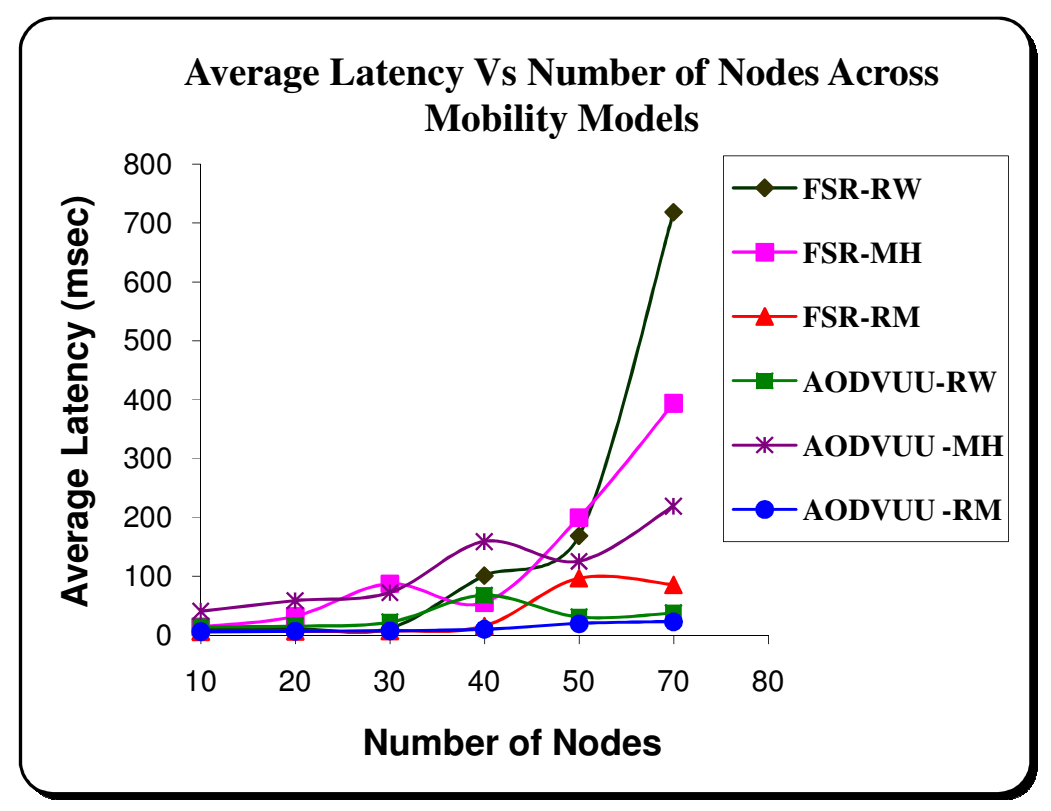

Figure 9. Number of Nodes Vs Average Latency Across three Mobility Models

In Figure 10, the network control overhead (NCO) for FSR and AODVUU across three mobility models is reported. From figure, it is observed that the network control overhead for both of the two protocols increases when the number of mobile hosts increases irrespective of the mobility model used. As FSR maintains the routing table for all the possible routes of the entire network, the network control overhead from the control messages is increasing while the number of the mobile hosts increasing. Again the overhead of reactive protocols like AODVUU is related mostly due to the discovery of new routes and from the updates of the usual routes.

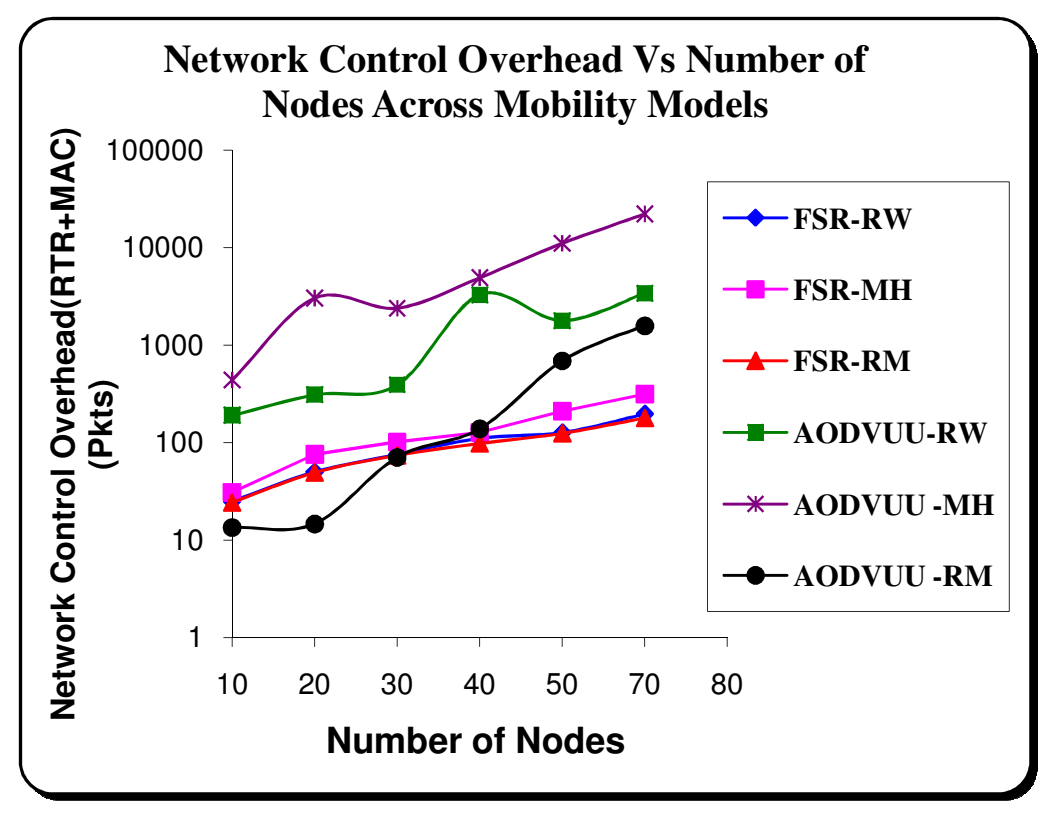

Figure 10. Number of Nodes Vs Network Control Overhead Across three Mobility Models 
As the undesirable environment for reactive protocols is the network with heavy traffic with large number of mobile hosts with high mobility, this situation will result that a big number of routes will break resulting repeated route discoveries and error reports in the network. Hence, AODVUU requires more control overhead at increasing network size.

It is also observed that the Reference Point Group mobility model is an optimum environment for both of the two protocols while Manhattan grid mobility model is more challenging. However, at Reference Point Group mobility model, the network control overhead for both FSR and AODVUU increases slowly for the increasing number of mobile hosts.

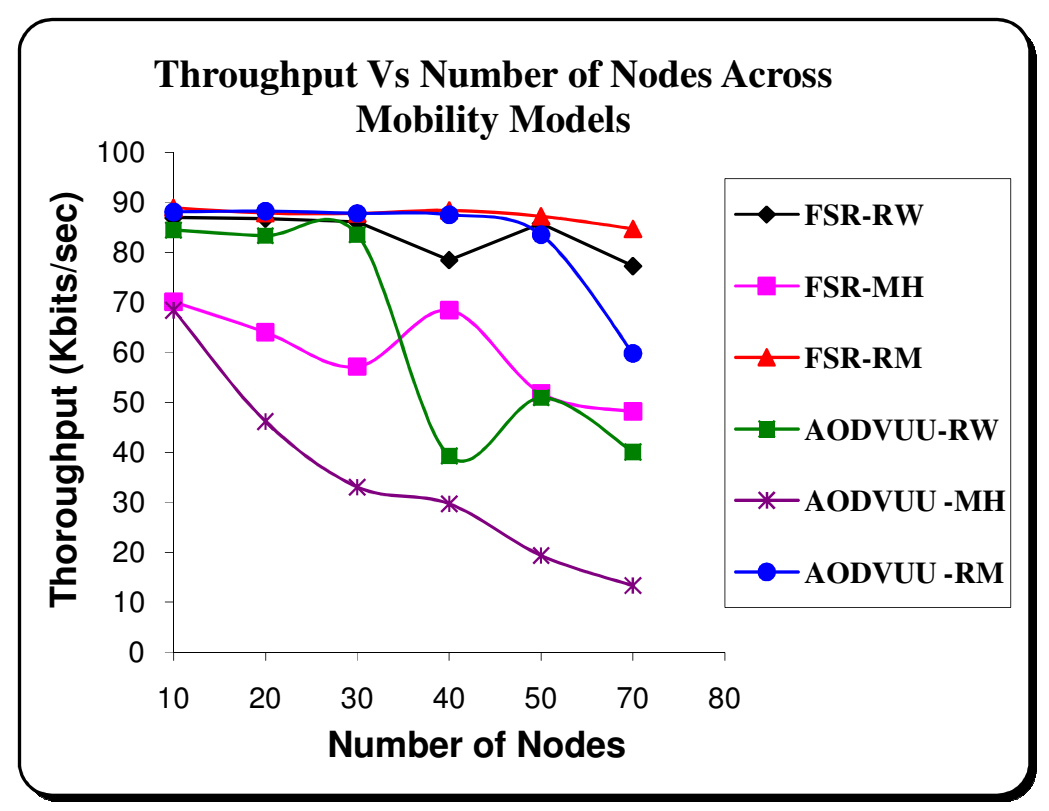

Figure 11. Number of Nodes Vs Throughput Across three Mobility Models

The results for throughputs for FSR and AODVUU are plotted in Figure 11, which is consistent with the result of network control overhead shown in Fig. 4.14. This is because the growth of overhead is directly related to the throughput drop. Results show that the throughputs for AODVUU degrades quickly than that of FSR when the number of nodes increases, no matter which mobility model is used. AODVUU's poor performance is due to heavy network control message overhead and hence the superiority of FSR is remarkable.

Again the throughput for FSR is sufficiently higher across Reference Point Group mobility model than other two mobility models. This is because the hierarchical structure of FSR can match well with the group motion. It is also noticed that the throughput for FSR Reference point group mobility model does not decrease too drastically when the number of nodes increases. This stability comes from the hierarchical routing structure. In terms of throughput, it is also observed that the Manhattan Grid - like street environment can be more challenging than the other two mobility models. 


\section{Conclusions}

This research studies and compares FSR and AODVUU routing protocols for MANETs. AODVUU uses a reactive and on-demand routing scheme whereas FSR is a proactive routing protocol, which uses "fisheye" technique to capture the update information of neighbors with high 'details'. The 'details' decreases as the distance increases. FSR is also an implicit hierarchical routing protocol.

Packet delivery ratio and average latency are very important when deciding how reliable a protocol is and how good it performs. Network control load is a measure of how effective a protocol is. A routing protocol must be both reliable and efficient when it is used in energyconstrained wireless networks with very limited bandwidth. In terms of packet delivery ratio, AODVUU performs better than FSR when there is low mobility. But FSR always outperforms AODVUU as host speed goes up across all three mobility models. Again both protocols show different results in different mobility models. Among three mobility models, Manhattan Grid mobility model shows abrupt results for both of these two routing protocols. At high data sending rate AODVUU performs better that FSR in terms of PDR and delay. On the other hand FSR outperforms AODVUU in terms of network control overhead and throughput with increasing data sending rate. At higher network load FSR is more scalable than AODVUU in all mobility models except Manhattan Grid environment. Also, proactive routing protocol FSR works most efficiently in the dense network. The following conclusions are drawn from the simulation results.

- FSR demonstrates sufficient reliability under high-speed environment.

- The performance of ad-hoc routing protocols is highly dependent on the mobility model used for the simulation.

- Reactive protocols are more speed-sensitive while proactive protocols are not.

- Routing in ad-hoc network over Manhattan Gird environments is more challenging than Random Waypoint and RPGM.

- In terms of PDR and delay, AODVUU outperforms FSR when data-sending rate is so high no matter which mobility model is used but FSR performs better than AODVUU for throughput and network control overhead.

- At dense network FSR is superior to AODVUU.

- Under Manhattan Grid mobility model, AODVUU shows better results when network load is high enough.

The recommendations for further study include:

- Energy conservation is an important issue in MANET because nodes often have finite battery power and the batteries will be drained and this event causes route breaks. Therefore, experiment should be extended to investigate the impact of the battery dying effect to the network performance.

- Security is an another key important issue in MANET hence experiment should be extended to address security issues with an extension of FSR and AODVUU which makes them more efficient and suitable for providing all requirements of mobile ad-hoc networks in the future work.

- The behaviour of FSR and AODVUU routing protocols can be studied with other mobility model such as Exponential Correlated Random Mobility Model, Pursue Mobility Model, A Boundless Simulation Area Mobility Model, Gauss-Markov Mobility Model and so on.

- Terrain models like hills, rivers, highways, urban roads, buildings, indoor partition layouts, etc can be accounted for, so that they both constrain node movements and influence the propagation models. 
International Journal of Computer Networks \& Communications (IJCNC) Vol.3, No.2, March 2011

\section{REFERENCES}

[1] Kunze, C., Grossmann, U., Storka, W., and Muller-Glaser, K., (2002), “Application of ubiquitous computing in personal health monitoring systems", In DGBMT: Jahrestagung der Deutschen Gesellschaft Für Biomedizinische Technik, pp. 360-362, 2002.

[2] Buszko, D., Lee, W., H., and Helal, A., (2001), "Decentralized ad-hoc groupware api and framework for mobile collaboration”, In GROUP'01: Proceedings of the International ACM SIGGROUP Conference on Supporting Group Work, pp. 5-14. ACM Press, 2001.

[3] Ritter, H., Tian, M., Voigt, T., and Jochen H. Schiller, J., H., (2003), “A highly flexible testbed for studies of ad-hoc network behaviour”, In LCN, pp. 746- 752, 2003.

[4] Görgen, D., Frey, H., and Hutter, C., (2005), "Information dissemination based on the en-passant communication pattern”, KiVS: Fachtagung Kommunikation in Verteilten Systemen, 2005.

[5] Riera, S., M., Wellnitz, O., and Wolf, L., (2003), "A zone-based gaming architecture for ad-hoc networks", In NETGAMES '03: Proceedings of the 2nd workshop on Network and system support for games, pp. 72-76. ACM Press, 2003.

[6] Frey, H., Lehnert, J., K., and Sturm, P., (2002), "Ubibay: An auction system for mobile multihop ad-hoc networks", In Workshop on Ad-hoc Communications and Collaboration in Ubiquitous Computing Environments, 2002.

[7] Belding-Royer, E., M., and Toh, C., K., (1999), “A review of current routing protocols for ad-hoc mobile wireless networks", IEEE Personal Communications Magazine, pp. 46-55, 1999

[8] Wikipedia, (2002), "Distance-vector routing protocol”, The free Encyclopedia, Electronic Doc. Available: http://en.wikipedia.org/wiki/Distancevector_routing_protocol, 18 Dec. 2002

[9] Laigar, P., (2002), "Analysis of Routing Algorithms for Mobile Ad-Hoc Networks", Thesis, Chalmers University of Technology, Department of Computer Engineering, Gothenburg 2002

[10] Broch, J., Maltz, D., Johnson, D., Hu, Y., and Jetcheva, J., (1998), "Multi-Hop Wireless Adhoc Network Routing Protocols", In Proceedings of the ACM/IEEE International Conference on Mobile Computing and Networking (MOBICOM), pages 85-97, 1998.

[11] Johnson, D., and Maltz, D., (1996), "Dynamic Source Routing in Ad Hoc Wireless Networks", In T. Imelinsky and H. Korth, editors, Mobile Computing, Kluwer Academic Publishers, pages 153-181, 1996.

[12] Camp, T., Boleng, J., and Davies, V., (2002), “A Survey of Mobility Models for Ad Hoc Network Research”, In Wireless Communication and Mobile Computing (WCMC), Vol. 2, No. 5, pages 483-502, 2002

[13] Tan, D.S., Zhou, S., Ho, J., Mehta, J.S., Tanabe, H., (2002), "Design and Evaluation of an Individually Simulated Mobility Model in Wireless Ad Hoc Networks", In Proc. Communication Networks and Distributed Systems Modelling and Simulation, San Antonio, TX, 2002

[14] Hong, X., Gelra, M., Pei, G., and Chiang, C., (1999), “A group mobility model for ad hoc wireless networks", In Proceedings of the ACM International Workshop on Modelling and Simulation of Wireless and Mobile Systems (MSWiM), Aug. 1999.

[15] Universal Mobile Telecommunications System (UMTS), (1998), "Selection Procedures for the Choice of Radio Transmission Technologies," of the UMTS (TS30.03 v3.2.0). TS 30.03 3GPP, April 1998.

[16] Broch, J., Maltz, D., A., Johnson, D., B., Hu, Y., C., and Jetcheva, J., (1998), “A Performance Comparison of Multi-Hop Wireless AD Hoc Network Routing Protocols", In Proceedings MobiCom'98, Dallas, TX, Oct. 1998.

[17] Pei, G., Gerla, M., and Chen, T., W., (2000), "Fisheye State Routing: A Routing Scheme for Ad Hoc Wireless Networks", In Proceedings of the IEEE International Conference on Communications, (ICC'00), Vol. 1, pp.70-74, New Orleans, June 2000. 
[18] Royer, E., M., Lee, S., J., and Perkins, C., E., (2000), "The effects of MAC protocol on ad hoc network communication", 2000.

[19] Raju, J., and Garcia-Luna-Aceves, J., J., (2000), “A comparison of on-demand and table driven routing for ad-hoc wireless networks," In Proceedings of IEEE International Conference on Communications, ICC'00, pp. 1702-1706 vol.3, New Orleans, Louisiana, June 2000.

[20] Lee, S., J., Hsu, J., Hayashida, R., Gerla, M., and Bagrodia, R., (2003), "Selecting a routing strategy for your ad hoc network”, In Computer Communications, vol. 26, pp. 723-733, 2003.

[21] Murthy, S., and Garcia-Luna-Aceves, J., J., (1996), “An efficient routing protocol for wireless networks," In ACM Mobile Networks and Applications Journal, Special issue on Routing in Mobile Communication Networks, 1996.

[22] Ko, Y., and Vaidya, N., H., (1998), "Location aided routing (LAR) mobile ad hoc networks," In MOBICOM, 1998.

[23] Basagni, S., Chlamtac, I., Syrotiuk, V., and Woodward, B., (1998), "A distance routing effect algorithm for mobility (DREAM),” In MOBICOM, pp. 76-84, 1998.

[24] Chen, B., and Chang, C., H., (2003), "Mobility Impact on Energy Conservation of Ad Hoc Routing Protocol”, White Papers, Published at Tufts University, May, 2003.

[25] Chen, J., Lee, Y., Z., Maniezzo, D., and Gerla, M., (2006), "Performance Comparison of AODV and OFLSR in Wireless Mesh Networks", In Proceedings of the fifth annual Mediterranean Ad Hoc Network, pp. 271-278, diit, Jiwei Chen, University of California at Los Angeles, US, 2006.

[26] Sirivianos, M., and Leontaris, A., (2006), "Comparative Evaluation of Ad-Hoc Routing Protocols in Highly Dynamic Environments”, University of California, Irvine, ICS, Technical Report 07-04, 2006.

[27] Linares, A., B., Karlsson, J., (2006), "Evaluation of TCP Performance in hybrid Mobile Ad Hoc Networks", Master's Thesis, Department of Computer Science, Karlstad University, Karlstad, 2006.

[28] Chen, T., W., and Gerla, M., (1998), "Global State Routing: A New Routing Scheme for Ad-hoc Wireless Networks”, In Proceedings of IEEE ICC'98, Atlanta, GA, pp. 171-175. Jun. 1998.

[29] Kleinrock, L., and Stevens, K., (1971), "Fisheye: A Lenslike Computer Display Transformation", Technical report, UCLA, Computer Science Department, 1971.

[30] Gerla, M., Hong, X., and Pei, G., (2002), "Fisheye State Routing Protocol (FSR) for Ad Hoc Networks", IETF Internet Draft (work in progress): draft-ieft- manet-fsr-03.txt, June 2002.

[31] Sun, A., C., (2000), "Desigh and Implementation of Fisheye Routing Protocol for Mobile Ad Hoc Networks", Master's thesis, Massachusetts Institute of Technology, May 2000.

[32] Perkins, C., Belding-Royer, E., Das, S., (2003), "Request for Comments: 3561", www.ietf.org/rfc/rfc3561.txt, Jul. 2003.

[33] Perkins, C., Belding-Royer, E., Das, S., (2002), “Ad hoc On-Demand Distance Vector (AODV) Routing”, Internet Draft, draft-ietf-manet-aodv-11.txt, work in progress, June 2002.

[34] NS2-URL, "The network simulator - NS2, http://www.isi.edu/nsnam/ns/"

[35] Tuch, B., (1993), "Development of WaveLAN, an ISM Band Wirelessw LAN", AT\&T Tech. J., vol. 72, no 4, July/Aug. 1993, pp. 27-33)(Lucent Technologies, http://www.lucent.com)

[36] Wall, C., de and Gerharz, M., (2003), "BonnMotion: a mobility scenario generation and analysis tool”, Communication Systems group, Institute of Computer Science IV, University of Bonn, Germany. Website: http://web.informatik.uni-bonn.de/IV/Mitarbeiter/dewaal/BonnMotion/, 2003.

[37] Chen, B., and Chang, C., H., (2003), "Mobility Impact on Energy Conservation of Ad Hoc Routing Protocol", White Papers, Published at Tufts University, May, 2003. 
[38] Wang, L., Shu, Y., Dong, M., Zhang, L., and Yang, W., W., (2001), “Adaptive Multipath Source Routing in Ad Hoc Networks”, IEEE International Conference on Communications, ICC 2001.

[39] Jayakumar, G., and Ganapathy, G., (2007), "Performance Comparison of Mobile Ad-hoc Netwrok Routing Protocol ”, International Journal of Computer Science and Netwrok Security (IJCSNS), VOL. 7 No. 11, Nov. 2007.

\section{Authors}

Foez Ahmed received the B. Sc. and M.Sc. Engineering degree in Information and Communication

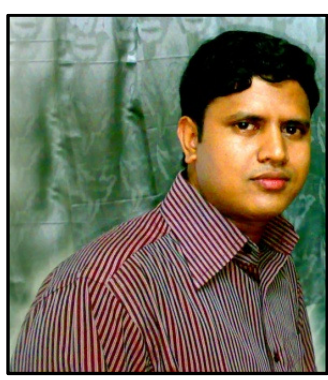
Engineering from Rajshahi University in 2007 and 2009 respectively. $\mathrm{He}$ joined as a Lecturer in the Dept. of Electronics and Communication Engineering, Northern University Bangladesh (NUB), Dhaka, Bangladesh in the 2008. At present he is on leave and working as a Lecturer with the Department of Computer Networks and Communication Engineering, College of Computer Science, King Khalid University, Kingdom of Saudi Arabia. He has received various Awards and Scholarships for the distinctive results. His research interests include Mobile Ad-hoc Networks and Routings, Cognitive Radio Networks, Cooperative Communications, Sensor Networks, Underwater Acoustic Networks, Wireless Body Area Networks and Cloud Computing.

Muhammad Sajjadur Rahim received the M. Engineering degree in Information Science and Systems Engineering from Ritsumeikan University, Japan in 2006 and the B. Sc. Engineering degree in Electrical

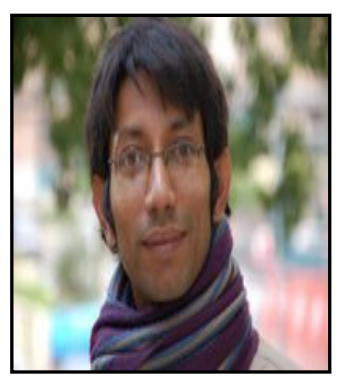
and Electronic Engineering from Bangladesh University of Engineering and Technology (BUET), Dhaka, Bangladesh in 1996. He had worked as a Faculty at NIIT, Beximco Systems Ltd., Dhaka, Bangladesh from September 1999 to December 2001. He then worked as an Executive in Engineering (Radio Planning) at Citycell (a mobile phone operator in Bangladesh) from January 2002 to August 2002. After that he joined as a Lecturer in the Department of Electronics and Communication Engineering, Khulna University, Khulna, Bangladesh from August 2002 to December 2002. At present, he is working as an Assistant Professor in the Department of Information and Communication Technology, University of Rajshahi since December 2002. Recently he is working towards his Ph.D. degree in Underwater Networks in the department of Information engineering at University of Padua, Italy since January 2009. His research interests include Mobile Ad-hoc Networks, Wireless Communication Systems \& Networks, Underwater Acoustic Communication Systems \& Networks, Delay-Tolerant Networking and Sensor Networks. 